\title{
Editors' Notice
}

We take pleasure in noting that the third Five College Regional Geometry Institute, funded by the National Science Foundation, to be held at Smith College during the month of July, 1993, will be devoted this year to discrete geometry.

There will be emphases on computational geometry and on tiling theory, with week-long lecture series in those areas given by Robert Connelly, John Conway, Ludwig Danzer, Scott Drysdale, Herbert Edelsbrunner, Joseph O'Rourke, Charles Radin, and Godfried Toussaint, as well as lectures and workshops on other areas such as polytopes, rigidity, discrete convexity, knot theory, combinatorial geometry, and arrangements. The tentative list of invited speakers includes, in addition to those already mentioned, Colin Adams, Imre Bárány, Louis Billera, Bernard Chazelle, H. S. M. Coxeter, Steve Fortune, Jacob E. Goodman, Leo Guibas, Ruth Haas, Daniel Huson, Wlodimierz Kuperberg, Jeff Lagarias, Carl Lee, Robert Meyerhoff, Victor Milenkovic, Frank Morgan, János Pach, Richard Pollack, Barry Schaudt, Thomas Schipper, Raimund Seidel, Micha Sharir, Steve Skiena, Diane Souvaine, Jean Taylor, Stan Wagon, Jeff Weeks, and Chee Yap.

The Institute, which will be run this year under the direction of Marjorie Senechal, is intended both to stimulate research in geometry and to encourage the mutual interaction of researchers, graduate students, high-school teachers, and undergraduates, centering around geometry as an area of common interest. Support will be offered to participants, who should contact the Regional Geometry Institute, c/o Department of Mathematics, Smith College, Northampton, MA 01063, USA (tel. (413) 585-2700, email rgi@smith.smith.edu) for application forms and for further information. The deadline for undergraduate REU applicants is February 15, 1993; for all others, March 30, 1993. 